\title{
ON STEADY INCOMPRESSIBLE THREE-DIMENSIONAL HYDROMAGNETIC FLOWS
}

\author{
G. PRASAD AND T. SINGH
}

\begin{abstract}
In this paper certain theorems of theoretical interest have been established with the help of the geometrical properties of Faraday's surface (which is spanned by the flow and field lines). These theorems shed light on the behaviour of steady incompressible hydromagnetic flows. The complex-lamellar acceleration and simple geodesic motion admitted by such flows have also been studied.
\end{abstract}

1. Introduction. In recent years, several works have been devoted to the study of geometrical aspects of hydromagnetic flows. Suryanarayan [1] has shown that Bernoulli's surfaces exist in the case of steady incompressible hydromagnetic flows when the magnetic field is in a fixed direction. This condition on the magnetic field leads directly to the conclusion that the Lorentz force is conservative. But this is not true in general. Wasserman [2] has shown that Faraday's equation for hydromagnetic systems with steady magnetic field ensures the existence of surfaces containing the flow and field lines. This result is interesting in the sense that the hydromagnetic flows with steady magnetic field do not admit the existence of surfaces, in general, except the surface spanned by the flow and field lines (which we call Faraday's surface for convenience). Thus it seems that the Faraday's surface plays a key role in the investigation of the geometrical aspects of such flows.

Purushotam and others [3-7] have also tried to study the geometrical properties of hydromagnetic flows without giving due importance to the existence of Faraday's surfaces.

The present investigation of geometrical properties of hydromagnetic flows begins with the consideration of Faraday's surfaces. We study some geometrical properties of this surface employing the techniques developed by Marris and Passman [8]. For simplicity, we consider incompressible infinitely conducting hydromagnetic system endowed with steady magnetic field and thereby establish some theorems on geometrical aspects of hydromagnetic flows. We also examine the possibility of complex lamellar acceleration and simple geodesic motion admitted by such flows.

2. Basic equations and geometric results. The basic equations governing steady incompressible hydromagnetic flows in the limit of infinite conductivity are [2]

$$
\nabla \cdot \vec{u}=0
$$

Received by the editors August 14, 1979 and, in revised form, May 28, 1981.

1980 Mathematics Subject Classification. Primary 53A17, 53B50; Secondary 76W05.

Key' words and phrases. Hydromagnetic flows, Faraday's surface, complex-lamellar acceleration, simple geodesic motion. 


$$
\begin{gathered}
\rho(\vec{u} \cdot \nabla) \vec{u}+\nabla p=\vec{J} \times \vec{h}, \\
\operatorname{curl}(\vec{u} \times \vec{h})=0, \\
\nabla \cdot \vec{h}=0,
\end{gathered}
$$

where $\vec{u}$ is the velocity vector, $p$ is the pressure, $\rho$ the density, $\vec{J}$ the current vector and $\vec{h}=\mu^{1 / 2} \vec{H}$, where $\mu$ is the magnetic permeability and $\vec{H}$ is the magnetic field vector.

We define a coordinate system on a sub-region $R$ of three dimensional Euclidean space $E_{3}$ as a set of ordered triples $x^{\alpha}(\alpha=1,2,3)$ such that there exists a one-one correspondence between the number $x^{\alpha}$ of the set and the points of $R$. Following Marris and Passman [8] we introduce the self-reciprocal basis consisting of orthogonal unit vectors $\hat{t}\left(x^{\alpha}\right), \hat{n}\left(x^{\alpha}\right), \hat{b}\left(x^{\alpha}\right)$ defining the tangent, principal normal and binormal to the given space curve through a point $x^{\alpha}$ of $E_{3}$. The results due to Marris and Passman [8] are

$$
\begin{gathered}
\operatorname{curl} \hat{t}=\Omega_{t} \hat{t}+k_{t} \hat{b}, \\
\operatorname{curl} \hat{n}=-(\operatorname{div} \hat{b}) \hat{t}+\Omega_{n} \hat{n}+\theta_{n t} \hat{b}, \\
\operatorname{curl} \hat{b}=\left(k_{t}+\operatorname{div} \hat{n}\right) \hat{t}-\theta_{b t} \hat{n}+\Omega_{b} \hat{b},
\end{gathered}
$$

where $k_{t}$ denotes the curvature of the space curve whose tangent is $\hat{t}$. The invariants $\Omega_{t}(=\hat{t} \cdot \operatorname{curl} \hat{t}), \Omega_{n}(=\hat{n} \cdot \operatorname{curl} \hat{n})$ and $\Omega_{b}(=\hat{b} \cdot \operatorname{curl} \hat{b})$ are, respectively, the abnormalities of the vector-lines of $\hat{t}, \hat{n}$, and $\hat{b} \cdot \theta_{n t}(=\hat{n} \cdot \operatorname{grad} \hat{t} \cdot \hat{n})$ and $\theta_{b t}$ $(=\hat{b} \cdot \operatorname{grad} \hat{t} \cdot \hat{b})$ are the normal deformations of the vector tube of $\hat{t}$ in the directions of $\hat{n}$ and $\hat{b}$ respectively. The commutation formulae [8] are

$$
\begin{gathered}
\delta_{b n}^{2} F-\delta_{n b}^{2} F=\Omega_{t} \delta_{t} F-\operatorname{div} \hat{b} \delta_{n} F+\left(k_{t}+\operatorname{div} \hat{n}\right) \delta_{b} F, \\
\delta_{t b}^{2} F-\delta_{b t}^{2} F=\Omega_{n} \delta_{n} F-\theta_{b t} \delta_{b} F, \\
\delta_{n t}^{2} F-\delta_{t n}^{2} F=k_{t} \delta_{t} F+\theta_{n t} \delta_{n} F+\Omega_{b} \delta_{b} F,
\end{gathered}
$$

where $F$ is a scalar point function. The symbols $\delta_{t}, \delta_{n}, \delta_{b}$ are used to denote the components $\hat{t} \cdot \operatorname{grad}, \hat{n} \cdot \operatorname{grad}, \hat{b} \cdot \operatorname{grad}$, respectively. An expression such as $\delta_{b n}^{2} F$ denotes $\hat{b} \cdot \operatorname{grad}(\hat{n} \cdot \operatorname{grad} F)$.

3. Faraday's surfaces in hydromagnetic flows. In this section we begin with the discussion of the geometrical properties of Faraday's surface. Let $\vec{u}=u\left(x^{\alpha}\right) \hat{t}\left(x^{\alpha}\right)$ be the velocity vector field for steady hydromagnetic motion, where $u$ is the velocity magnitude and $\hat{t}$ the unit vector tangential to the flow line. The unit vector $\hat{n}$ points along the principal normal to the flow line and $\hat{b}$ is its unit binormal. We can define the magnetic field $\vec{h}$ in terms of the flow line triad as

$$
\vec{h}=h_{t} \hat{t}+h_{n} \hat{n}+h_{b} \hat{b},
$$

where $h_{t}, h_{n}$ and $h_{b}$ denote, respectively, the tangential, principal normal and binormal components of magnetic field $\vec{h}$. From (2.3), (3.1) and (2.5)-(2.7) we obtain

$$
\delta_{n}\left(u h_{n}\right)+\delta_{b}\left(u h_{b}\right)+u h_{b} \operatorname{div} \hat{b}+u h_{n}\left(k_{t}+\operatorname{div} \hat{n}\right)=0,
$$




$$
\begin{aligned}
& \delta_{t}\left(u h_{n}\right)+u h_{n} \theta_{b t}+u h_{b} \Omega_{n}=0, \\
& u h_{n} \Omega_{b}-\delta_{t}\left(u h_{b}\right)-u h_{b} \theta_{n t}=0 .
\end{aligned}
$$

We now state the following result:

THEOREM (3.1). The flow lines are geodesics on Faraday's surfaces if the principal normal component of magnetic field vanishes.

Proof. Since Faraday's surfaces are spanned by the flow and field lines, the vanishing of the principal normal component of magnetic field implies that the field lines are normal to the principal normals of the flow lines. Thus the principal normals of the flow lines are normal to the Faraday's surfaces and hence the flow lines are geodesics.

THEOREM (3.2). For steady incompressible hydromagnetic flows with vanishing $h_{n}$, the family of Faraday's surfaces form a family of developables iff $\delta_{t}\left(h_{b}\right)$ is constant along the flow lines.

Proof. Since $h_{n}=0$, it follows from (3.2) and (3.4) that

$$
\operatorname{div} \hat{b}=-\delta_{b}\left(\ln u h_{b}\right) \text {, }
$$

and

$$
\theta_{n t}=-\delta_{t}\left(\ln u h_{b}\right) \text {. }
$$

By Theorem (3.1), the flow lines are geodesics on Faraday's surface. Since $\hat{b}$ is orthogonal to $\hat{n}$ and lies in the tangent plane to Faraday's surface, the vector-lines of $\hat{b}$ will be orthogonal to geodesic flow lines. Hence the vector-lines of $\hat{b}$ are parallel curves on Faraday's surface. According to Marris [9] the $t$-lines and $b$-lines form an allowable coordinate system on a small region of Faraday's surface. In this coordinate system the first fundamental form of Faraday's surface is given by

$$
d \sigma^{2}=d t^{2}+g(t, b) d b^{2},
$$

where the metric element $g(t, b)$ is related to the Gaussian curvature $G$ of Faraday's surface by the relation [9]

$$
G=-\frac{1}{g^{1 / 2}} \delta_{t}^{2}\left(g^{1 / 2}\right)
$$

with

$$
\delta_{t}\left(\ln g^{1 / 2}\right)=\theta_{b t}
$$

From (2.1), we obtain

$$
\delta_{t}(\ln u)+\theta_{n t}+\theta_{b t}=0 .
$$

It follows from (3.6) and (3.10) that

$$
\theta_{b t}=\delta_{t}\left(\ln h_{b}\right) .
$$

From (3.9) and (3.11), we have

$$
g^{1 / 2}=f(b) h_{b}
$$


where $f$ is an arbitrary function of $b$. By a change of scale in the $b$-direction we can reduce $(3.12)$ to

$$
g^{1 / 2}=h_{b}
$$

The substitution of (3.13) into (3.8) yields

$$
G=-\left(1 / h_{b}\right) \delta_{t}^{2}\left(h_{b}\right)
$$

This shows that the Gaussian curvature of Faraday's surfaces vanishes iff $\delta_{t}\left(h_{b}\right)$ is constant along the flow lines.

4. Noncirculation preserving hydromagnetic flows with complex-lamellar acceleration. This section is devoted to the discussion of the noncirculation preserving hydromagnetic motion with complex-lamellar acceleration. It is easy to write (2.2) in the form

$$
\rho \vec{a}+\nabla p=\vec{J} \times \vec{h},
$$

where $\vec{a}$ is the acceleration vector given by

$$
\vec{a}=\delta_{t}\left(\frac{1}{2} u^{2}\right) \hat{t}+k_{t} u^{2} \hat{n}=a_{t} \hat{t}+a_{n} \hat{n} .
$$

Operating curl on both sides of (4.1), we get

$$
\rho \operatorname{curl} \vec{a}=\operatorname{curl}(\vec{J} \times \vec{h}) .
$$

Since the Lorentz force $(\vec{J} \times \vec{h})$ is nonconservative, $\operatorname{curl}(\vec{J} \times \vec{h})$ cannot vanish. This means that there is no possibility of circulation preserving hydromagnetic motion (except the case of force-free motion). But this does not eliminate the possibility of hydromagnetic motion with complex-lamellar acceleration. As we know that the motion is said to be endowed with complex-lamellar acceleration iff the acceleration vector satisfies the condition [9]

$$
\vec{a}=\xi \operatorname{grad} \eta,
$$

where $\xi$ and $\eta$ are scalar point functions. It can be easily shown from (4.3) and (4.4) that $\operatorname{curl}(\vec{J} \times \vec{h}) \neq 0$. From (4.4) we have

$$
\vec{a} \cdot \operatorname{curl} \vec{a}=0 \text {. }
$$

From (4.3) and (4.5)

$$
\vec{a} \cdot \operatorname{curl}(\vec{J} \times \vec{h})=0 .
$$

This may be regarded as a necessary and sufficient condition for the hydromagnetic flows admitting complex-lamellar acceleration.

On assuming that the principal normal component of the magnetic field vanishes, we may rewrite (3.1) as

$$
\vec{h}=h_{t} \hat{t}+h_{b} \hat{b}
$$

From (2.5), (2.7) and (4.7), we obtain

$$
\vec{J}=J_{t} \hat{t}+J_{n} \hat{n}+J_{b} \hat{b}
$$

where

$$
J_{t}=h_{t} \Omega_{t}+\delta_{n}\left(h_{b}\right)+h_{b}\left(k_{t}+\operatorname{div} \hat{n}\right)
$$




$$
\begin{gathered}
J_{n}=\delta_{b}\left(h_{t}\right)-\delta_{t}\left(h_{b}\right)-h_{b} \theta_{b t}, \\
J_{b}=h_{b} \Omega_{b}+h_{t} k_{t}-\delta_{n}\left(h_{t}\right) .
\end{gathered}
$$

It follows from (2.5)-(2.7), (3.5), (3.6), (3.11), (4.7) and (4.8) that

$$
\begin{aligned}
\operatorname{curl}(\vec{J} \times \vec{h})= & {\left[h_{b} J_{n} \Omega_{t}-\delta_{b}\left(h_{t} J_{b}-h_{b} J_{n}\right)+\left(h_{t} J_{b}-h_{b} J_{t}\right) \delta_{b}\left(\ln u h_{b}\right)\right.} \\
& \left.-\delta_{n}\left(h_{t} J_{n}\right)-h_{t} J_{n}\left(k_{t}+\operatorname{div} \hat{n}\right)\right] \hat{t} \\
& +\left[\delta_{b}\left(h_{b} J_{n}\right)+\delta_{t}\left(h_{t} J_{n}\right)+h_{t} J_{n} \delta_{t}\left(\ln h_{b}\right)\right] \hat{n} \\
& +\left[h_{b} J_{n} k_{t}-h_{t} J_{n} \Omega_{b}-\delta_{n}\left(h_{b} J_{n}\right)-\left(h_{t} J_{b}-h_{b} J_{t}\right) \delta_{t}\left(\ln u h_{b}\right)\right] \hat{b},
\end{aligned}
$$

where the condition $\Omega_{n}=0$ is invoked. We now establish the following theorem.

THEOREM (4.1). A steady incompressible rotational noncirculation preserving hydromagnetic motion for which $h_{n}=\delta_{b}(p)=0$ and none of $a_{t}, k_{t}, h_{t}$ vanishes is endowed with complex-lamellar acceleration iff one of the following holds:

(i) $\delta_{b}\left(\ln u h_{b}\right)=0$,

(ii) $\Omega_{t}=\left(a_{n} / a_{t}\right) \delta_{b}\left[\ln \left(a_{n} / u h_{b}\right)\right]$.

Proof. From (4.1), (4.2), (4.7) and (4.8), we have

$$
\begin{gathered}
\rho a_{t}+\delta_{t} p=h_{b} J_{n}, \\
\delta_{b} p=-h_{t} J_{n}, \\
\rho k_{t} u^{2}+\delta_{n} p=h_{t} J_{b}-h_{b} J_{t} .
\end{gathered}
$$

From (4.14), since $\delta_{b} p=0$ and $h_{t} \neq 0$, it follows that $J_{n}=0$. Then from (4.2), (4.6) and (4.12), we get

$$
a_{t}\left(J_{t} h_{b}-h_{t} J_{b}\right) \delta_{b}\left(\ln u h_{b}\right)=0 .
$$

From (4.15) and (4.16),

$$
\left(\rho k_{t} u^{2}+\delta_{n} p\right) \delta_{b}\left(\ln u h_{b}\right)=0
$$

This leads to

$$
\delta_{b}\left(\ln u h_{b}\right)=0,
$$

or

$$
\rho k_{t} u^{2}+\delta_{n} p=0 .
$$

Equation (4.18a) is the first required condition. Differentiating (4.18b) along $\hat{b}$, we get

$$
\rho\left[\left(\delta_{b} k_{t}\right) u^{2}+2 u k_{t} \delta_{b} u\right]+\delta_{b n}^{2} p=0 .
$$

The use of (2.8) for $p$ reduces (4.19) to

$$
\rho\left[\left(\delta_{b} k_{t}\right) u^{2}+2 u k_{t} \delta_{b} u\right]+\Omega_{t} \delta_{t} p-\operatorname{div} \hat{b} \delta_{n} p=0 .
$$

It follows from (3.5), (4.2), (4.13), (4.18b), (4.20) and the condition $J_{n}=0$ that

$$
\Omega_{t}=\left(a_{n} / a_{t}\right) \delta_{b}\left[\ln \left(a_{n} / u h_{b}\right)\right] .
$$

This is the second condition stated in the theorem. 
THEOREM (4.2). A steady incompressible rotational noncirculation preserving hydromagnetic motion is endowed with complex-lamellar acceleration iff $\left[\left(1 / u h_{b}\right)\left(a_{n} / a_{t}\right)+\right.$ $\psi$ ] is constant along $b$-lines on Faraday's surface, where $\partial \psi / \partial b=-\Omega_{t} / u$.

The proof of this theorem is omitted because it follows easily from the results of Marris [9].

5. Noncirculation preserving hydromagnetic flows with simple geodesic motion. In this section we begin with the definition of simple geodesic motion. According to Marris [9], a steady, rotational, nonrectilinear, nonplane motion whose stream-line geometry satisfies the condition

$$
\Omega_{n}=\hat{n}, \quad \operatorname{curl} \hat{n}=0,
$$

is called a goedesic motion. A geodesic motion obeying the condition

$$
\omega_{n}=0 \Leftrightarrow \hat{n} \cdot \vec{\omega}=0
$$

is called simple geodesic motion. In order to discuss simple geodesic hydromagnetic motion we first establish the following result.

THEOREM (5.1). For a steady incompressible noncirculation preserving hypermagnetic flow with $h_{n}=0=\operatorname{div}\left(h_{b} \hat{b}\right)$, there exists a family of Lamb surfaces.

Proof. Since $h_{n}=0,(3.2)$ reduces to

$$
\delta_{b}\left(u h_{b}\right)+u h_{b} \operatorname{div} \hat{b}=0 .
$$

The expression for vorticity is [8]

$$
\vec{\omega}=\omega_{t} \hat{t}+\omega_{n} \hat{n}+\omega_{b} \hat{b}
$$

where

$$
\omega_{t}=\Omega_{t} u, \quad \omega_{n}=\delta_{b} u, \quad \omega_{b}=\left(k_{t} u-\delta_{n} u\right) .
$$

The use of (5.5) in (5.3) gives

$$
h_{b} \omega_{n}+u \operatorname{div}\left(h_{b} \hat{b}\right)=0 .
$$

Since $\operatorname{div}\left(h_{b} \hat{b}\right)=0,(5.6)$ reduces to $\omega_{n}=0$. This together with (5.4) leads to

$$
\vec{\omega} \times \vec{u}=u \omega_{b} \vec{n} \text {. }
$$

It follows from (5.7) that

$$
(\vec{\omega} \times \vec{u}) \cdot \operatorname{curl}(\vec{\omega} \times \vec{u})=\left(u \omega_{b}\right)^{2} \Omega_{n} .
$$

Since $h_{n}=0$ (or $\Omega_{n}=0$ ), (5.8) becomes the necessary and sufficient condition for the existence of a family of Lamb surfaces. This completes the proof of the theorem.

REMARK. Since the normal to the Faraday's surface coincides with that of the Lamb surface, the levels of these two surfaces coincide. Thus the Lamb surface and Faraday's surface are the same in this case.

COROllary (5.1). A steady incompressible hydromagnetic flow admits a simple geodesic motion if the flow is such that $h_{n}=0=\operatorname{div}\left(h_{b} \hat{b}\right)$. 
Proof. Since the vanishing of $h_{n}$ and $\operatorname{div}\left(h_{b} \hat{b}\right)$ implies the vanishing of $\Omega_{n}$ and $\omega_{n}$ respectively, it follows from (5.1) and (5.2) that the hydromagnetic flows admit simple geodesic motion. Further from (3.13) and (5.4),

$$
\begin{aligned}
\operatorname{curl}(\vec{\omega} \times \vec{u})= & u\left[\frac{1}{u h_{b}^{2}} \delta_{t}\left(u h_{b}^{2} \omega_{t}\right)-2 k_{t} \omega_{n}\right] \hat{t}+\frac{1}{h_{b}} \delta_{t}\left(h_{b} u \omega_{n}\right) \hat{n} \\
& +\left[\frac{1}{u h_{b}} \delta_{t}\left(u^{2} h_{b} \omega_{b}\right)-\Omega_{b} \omega_{n}\right] \hat{b}
\end{aligned}
$$

where the condition $\Omega_{n}=0$ has been used. We now establish the following two theorems for simple geodesic hydromagnetic motion.

THEOREM (5.2). A steady incompressible noncirculation preserving hydromagnetic flow has simple geodesic motion iff there exists a potential function $\xi(t, b)$ such that

$$
h_{t} h_{b} J_{n}=-\frac{\partial \xi}{\partial b} \text { and } h_{b} J_{n}=\frac{\partial \xi}{\partial t}
$$

on the Faraday's surface.

Proof. For simple geodesic motion, it follows from (5.2) and (5.9) that

$$
\hat{n} \cdot \operatorname{curl}(\vec{\omega} \times \vec{u})=0 .
$$

From (4.3), (4.12) and (5.10), we obtain

$$
\delta_{b}\left(h_{b} J_{n}\right)+\delta_{t}\left(h_{t} J_{n}\right)+h_{t} J_{n} \delta_{t}\left(\ln h_{b}\right)=0 .
$$

This may also be written as

$$
\frac{\partial}{\partial t}\left(h_{t} h_{b} J_{n}\right)+\frac{\partial}{\partial b}\left(h_{b} J_{n}\right)=0
$$

where

$$
\delta_{t}=\frac{\partial}{\partial t}, \quad h_{b} \delta_{b}=\frac{\partial}{\partial b}
$$

By the theorem of vector potential there must exist a function $\xi(t, b)$ such that

$$
h_{t} h_{b} J_{n}=-\frac{\partial \xi}{\partial b}, \quad h_{b} J_{n}=\frac{\partial \xi}{\partial t} .
$$

This proves the statement.

TheOREM (5.3). A simple geodesic hydromagnetic motion, for which $\delta_{t}(u) \neq 0$, admits complex-lamellar acceleration iff $\left(u h_{b}^{2} \omega_{t}\right)$ is constant along the flow lines on the Faraday's surface.

The proof of this theorem follows from (4.5), (5.2), (5.9) and (5.10).

ACKNOWLEDGment. We are thankful to the referee who gave us some most helpful advice for the revision of this paper.

\section{REFERENCES}

1. E. R. Suryanarayan, On the geometry of streamlines in hydromagnetic fluid flows when the magnetic field is along a fixed direction, Proc. Amer. Math. Soc. 16 (1965), 90-96.

2. R. H. Wasserman, On the geometry of magnetohydrodynamic flows, Quart. J. Mech. Appl. Math. 20 (1967), 219. 
3. G. Purushotham, On the geometry of streamlines in hydromagnetic fluid flows. Tensor (N. S.) 25 (1972), 229-237.

4. G. Purushotham and S. S. Rao, On complex-lamellar hydromagnetic steady gas flows, Tensor (N. S.) 20 (1969), 343-346.

5. E. R. Suryanarayan, Intrinsic equations for hydromagnetic flows, Rev. Roumaine Math. Pures Appl. 17 (1972), 103-112.

6. A. Indrasena. Steady rotating hydromagnetic flows, Tensor (N. S.) 32 (1978), 350-354.

7. G. Prasad, S. S. Gangwar and S. B. Misra, Intrinsic equations for the magnetofluid flows, Indian J. Pure Appl. Math. 10 (1979), 204-208.

8. A. W. Marris and S. L. Passman, Vector fields and flows on developable surfaces, Arch. Rational Mech. Anal. 32 (1969), 29-86 and the references quoted therein.

9. A. W. Marris, On steady three-dimensional motions, Arch. Rational Mech. Anal. 35 (1969), 122-168.

Department of Mathematics, S. C. College, Ballia-277001, India

Applied Mathematics Section, Institute of Technology, Banaras Hindu University, VARANASI-221005, INDIA 